\title{
Motion Planning for Archwire Bending Robot in Orthodontic Treatments
}

\author{
Zhang Yongde ${ }^{1,}$, , Wei Chunge ${ }^{1}$, Jiang Jingang ${ }^{1, b}$, Jiang Jixiong ${ }^{1}$, Liu $\mathrm{Yi}^{2}$ and Wang \\ Yong $^{2}$ \\ ${ }^{1}$ Intelligent Machine Institute, Harbin University of Science and Technology, China \\ ${ }^{2}$ Peking University School of Stomatology, China \\ azhangyd@hrbust.edu.cn, ${ }^{b}$ jiangjingang@hrbust.edu.cn
}

\begin{abstract}
This paper presents a motion planning method for archwire bending robot in orthodontic treatments. Because the movement of the archwire bending robot belongs to typical hybrid point-to-point movement, which has both long and short distance, thus the traditional acceleration/deceleration (acc/dec) method is not suitable for this mode of motion. In order to improve the stability and relief the impact and vibration in the process of archwire bending, a new motion planning method that based on three-order pure $S$ acc/dec curve, 5 segments acc/dec curve, and modified acc/dec curve is established and a piecewise self-adjustment acc/dec strategy that based on displacements of three-order pure $S$ acc/dec curve, 5 segments acc/dec curve, and modified acc/dec curve is proposed. The effect of acc/dec methods on the process of archwire bending is analyzed, and verification of the proposed method is conducted through MATLAB simulation. The MATLAB simulation realized validity of the motion planning.
\end{abstract}

Keywords: Motion planning, Acceleration and Deceleration, Orthodontic, Archwire bending robot

\section{Introduction}

Malocclusion is one common oral disease, as one of the three major oral diseases by World Health Organization. According to statistics, about $67.82 \%$ of children and young people have some degrees of malocclusion in China. Currently the best way to treat malocclusion is to wear archiwre. Through the archwire generated successive and a certain value of elastic force during the deformation to control the movement of the teeth, the purpose of correction can be achieved [1].At present, bending the orthodontic archwire is mostly done by hand, the process is not only inefficient, labor-intensive, and accuracy not guaranteed, but also prone to the archwire breakage. A reasonable way to solve these problems mentioned is to use robots to replace manual bending. The research on archwire bending robot has been lasted few years. What's more, as for the robot motion planning is a very important part of the research.

Point-to-point movement is a basic and important motion method of robot, the same as the archwire bending robot, which belongs to the typical hybrid point-point motion. There are both large motion displacement and small motion displacement between control points. The control points and displacements based on mathematical model of basic archiwire curve are shown in Figure 1. As a result of this motion method, it has obvious start-stop movement. If motion planning is not reasonable, obvious impact and vibration will be generated, and the end actuator positioning inaccuracy will be bonded to. Thus it is necessary to study on the acc/dec method between two control points for the robot, and make the robot in the process of 
running with shortest time and precision of the optimal effect. Linear acc/dec, exponential acc/dec, S curve acc/dec, and trigonometric acc/dec are the most common acc/dec methods. However, each of them has its own limitation [2-4]. Much deeper research is devoted to these methods by many researchers.

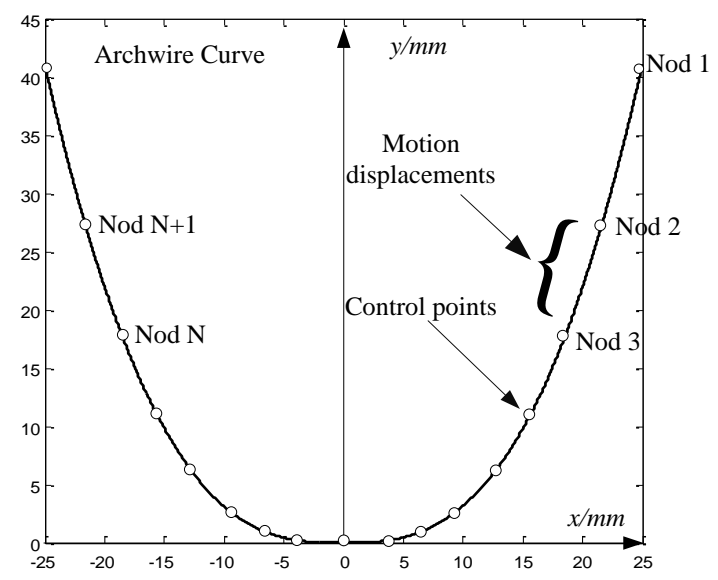

Figure 1. Control Points and Motion Displacements of Archwire Bending Robot

Some researchers proposed new acc/dec planning based on $\mathrm{S}$ curve [5-11]. Tian et al., proposed an improved S-shape acc/dec algorithm, which could quickly figure out the run time of the various stages [12]. And some researchers proposed acc/dec planning method by NURBS curvilinear interpolation [13-15]. Rew, Wook et al., proposed analytic equations for generating motion contour [16-17].

This paper presents a motion planning method for archwire bending robot in orthodontic treatments. The advantage is that the motion planning can ensure the archwire bending robot move to each control point smoothly at higher efficiency, and the running time is more optimal with piecewise self-adjusting distribution strategy of acc/dec. Three-order pure $S$ acc/dec curve, 5 segments $\mathrm{S}$ acc/dec curve, and modified three-order pure $\mathrm{S}$ acc/dec curve are established as the target curve.

The rest of this paper is organized as follows. Section 2 describes the robot model. Section 3 formulates the motion planning. Section 4 presents the process of motion planning simulated by MATLAB for archwire bending robot, and the result from the simulation verify the practicality of this motion planning. Experimental results are carried out in Section 5 while concluding remarks are given in Section 6 to close this paper.

\section{Archwire Bending Robot Model Description}

As is shown in Figure 2, IMI-1 type of orthodontic archwire bending robot is developed by Intelligence Machine Institute, Harbin University of Science and Technology. The robot is composed of three parts. The first part is the robot body that based on Cartesian coordinate scheme, it bends the archwire into shape; the second part is the hardware of the robot control system, it is used for completing complex program planning, preparation of motion programs, implementation of the bending device drivers and other tasks; the third one is the software of the robot control system, which is used for developing the control interface and compiling individual configuration file for the motion axes and controllers. In the process of archwire bending, an important way to ensure the accuracy of the processing is to guarantee the accurate positioning 
with high efficiency. One requirement for a good acc/dec method is to keep mechanism smoothly, steady and rapidly while working. If motion planning is not reasonable, it could generate obvious impact and vibration easily during start-stop situations, and bound to cause the end actuator positioning inaccuracy. Meanwhile, in order to reduce impact and vibration, we need to increase the stable time during the vibration; In other words, the mechanism cannot do any movement without increasing the total time.

The motion method of archwire bending robot belongs to the typical hybrid point-topoint movement, and the rate of movement in each control point is zero, so more start stop times are added. In order to ease the impact and vibration, the following three options are proposed: (1) Improve the accuracy of machining, and guarantee the accuracy of the assembly; (2) Selected reasonable acc/dec method; (3) Increase stability time during vibration. However, scheme (3) will increase the time of the whole process of bending the archwire, so the scheme (3) is ruled out.

At present, there are many ways concerning the acc/dec method, such as the linear acc/dec method, and exponential acc/dec method. These methods belong to secondorder acc/dec method, although ensure fast, the motion can also exert significant impact and vibration. If using the general 7 segments $S$ curve acc/dec method, which can only ensure the accurate location in long distance; when under the short distance movement, because of the speed and acceleration cannot reach the maximum, resulting in uniform segment during the running does not appear, which will also exert significant impact and vibration. While using the $S$ curve to determine the general condition of the 7 segments is too complicated, and the implement of the program algorithm is also more difficult, and real-time is not easy to guarantee. Meanwhile, according to the movement characteristics of the archwire bending robot, because the movement distance has the branch length, the effect should not be ideal if we use the acc/dec method mentioned directly.

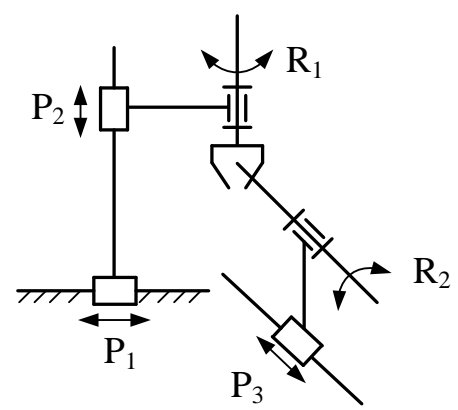

(a) Structure diagram

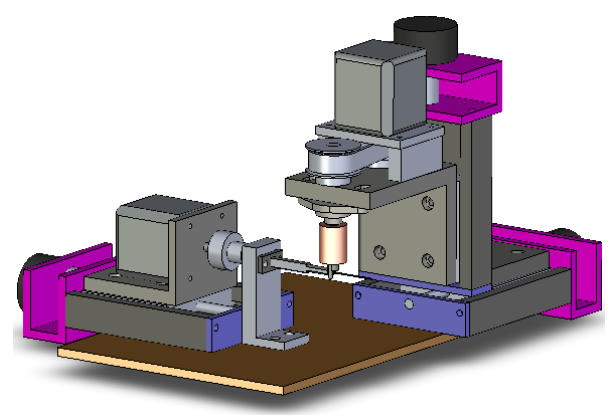

(b) 3-Dimension structure

Figure 2. IMI-1 type of Orthodontic Archwire Bending Robot Structure Diagram

\section{Motion Planning Formulation}

For the characteristics of robot motion, a piecewise self-adjusting distribution strategy of acc/dec is proposed. Combined with the system parameters of the archwire bending robot, three-order pure $\mathrm{S}$ acc/dec curve, 5 segments acc/dec curve and modified $\mathrm{S}$ acc/dec curve are established. And based on the displacement of three-order pure $\mathrm{S}$ acc/dec curve, a piecewise self-adjusting judgment is proceeded by the distance between the control points planned. If the distance is larger than the displacement of three-order pure $S$ curve, adopt 5 segments acc/dec curve with uniform velocity, or else adopt modified $\mathrm{S}$ acc/dec curve. 


\subsection{Establishment of Three-order Pure S Acc/Dec Curve}

We define the maximum jerk is $j_{\max }$, the maximum acceleration is $a_{\max }$, and the maximum velocity is $v_{\max }$, which are the system parameters of the archwire bending robot. The process of establishing three-order pure $\mathrm{S}$ acc/dec curve is described as follows. Make jerk be the maximum, and acceleration changes according to the jerk. The acceleration immediately decreases to zero while increases to the maximum. Symmetrically, the acceleration decreases to zero while increases to the maximum in opposite position with a constant maximum jerk. At this time, the velocity falls to zero from maximum. The establish jerk, velocity, acc/dec and displacement are shown in Figure 3.

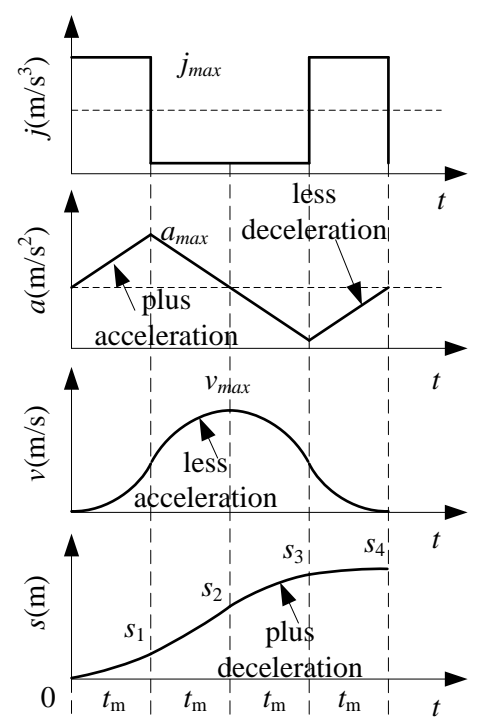

Figure 3. Three-order Pure S acc/dec Curve

In Figure 3, we assume the displacement of three-order pure $\mathrm{S}$ acc/dec curve is $s^{*}$. If the movement distance is longer than $s^{*}$, we call it long motion distance session, otherwise short motion distance session.

Because of the process time of pure S plus acc/dec and less acc/dec are completely the same, and the processes of velocity increase and decrease are totally symmetrical. Therefore, we assume the time of plus acceleration, less acceleration, plus deceleration and less deceleration is $t_{\mathrm{m}}$, which are all the same. The displacement of plus acceleration segments is $s_{1}$; the sum of displacement of less acceleration segments and plus acceleration segments is $s_{2}$; the sum of displacement of plus deceleration segments, plus deceleration segments and plus acceleration segments is $s_{3}$; the sum of displacement of less deceleration and $s_{3}$ is $s_{4}$. Hence, the expressions of three-order pure $\mathrm{S}$ acc/dec are shown in Equations (1)-(4).

$$
\left\{\begin{array}{l}
a_{1}=j_{\max } \cdot t \\
v_{1}=v_{0}+\frac{1}{2} j_{\max } \cdot t^{2}, \quad t \in\left[0, t_{m}\right] \\
s_{1}=v_{0} \cdot t+\frac{1}{6} j_{\max } \cdot t^{3}
\end{array}\right.
$$




$$
\begin{aligned}
& a_{2}=j_{\text {max }} \cdot t_{m}-j_{\text {max }} \cdot\left(t-t_{m}\right) \\
& \left\{v_{2}=v_{1}+j_{\text {max }} \cdot t_{m} \cdot\left(t-t_{m}\right)-\frac{1}{2} j_{\text {max }} \cdot\left(t-t_{m}\right)^{2} \quad, \quad t \in\left[t_{m}, 2 t_{m}\right]\right. \\
& s_{2}=s_{1}+v_{1} \cdot\left(t-t_{m}\right)+\frac{1}{2} j_{\max } \cdot t_{m} \cdot\left(t-t_{m}\right)^{2}-\frac{1}{6} j_{\max } \cdot\left(t-t_{m}\right)^{3} \\
& a_{3}=-j_{\text {max }} \cdot\left[t-2 t_{m}\right] \\
& \left\{v_{3}=v_{2}-\frac{1}{2} j_{\max } \cdot\left[t-2 t_{m}\right]^{2} \quad, \quad t \in\left[2 t_{m}, 3 t_{m}\right]\right. \\
& s_{3}=s_{2}+v_{2} \cdot\left[t-2 t_{m}\right]-\frac{1}{6} j_{\text {max }} \cdot\left[t-2 t_{m}\right]^{3} \\
& a_{4}=-j_{\text {max }} \cdot t_{m}+j_{\text {max }} \cdot\left[t-3 t_{m}\right] \\
& \left\{v_{4}=v_{3}-j_{\text {max }} \cdot t_{m} \cdot\left[t-3 t_{m}\right]+\frac{1}{2} j_{\text {max }} \cdot\left[t-3 t_{m}\right]^{2} \quad, \quad t \in\left[3 t_{m}, 4 t_{m}\right]\right. \\
& s_{4}=s_{3}+v_{3} \cdot\left[t-3 t_{m}\right]-\frac{1}{2} j_{\max } \cdot t_{m} \cdot\left[t-3 t_{m}\right]^{2}+\frac{1}{6} j_{\max } \cdot\left[t-3 t_{m}\right]^{3}
\end{aligned}
$$

\subsection{Establishment of 5 Segments Acc/Dec Curve}

Based on the three-order pure $\mathrm{S}$ acc/dec curve, when the movement distance is longer, in order to reduce the residual impact and vibration, and improve efficiency, velocity curve should exist in the system maximum velocity of uniform motion phase. In the meantime, the time in uniform motion phase should be longer to reduce the system running time. So the creation establishment of acceleration curve is that the acceleration increases to maximum by the maximum jerk, then decreases to zero in the same way, and keep this status for a while. In the end, the velocity falls to zero in the way as acceleration process. The established jerk, velocity, acc/dec and displacement are shown in Figure 4.

The expressions of 5 segments acc/dec are shown in Equations (6)-(10). Where the time of uniform motion is $t_{\mathrm{c}}$, the definition of $t_{\mathrm{m}}$ is the same as in pure $\mathrm{S}$ acc/dec curve. We can see from the Figure 4 and expressions that if $t_{\mathrm{c}}$ and $t_{\mathrm{m}}$ are confirmed in long distant motion, the expressions of acc/dec are determined.

$$
\left\{\begin{array}{l}
a_{1}=j_{\text {max }} \cdot t \\
v_{1}=v_{0}+\frac{1}{2} j_{\text {max }} \cdot t^{2} \quad, \quad t \in\left[0, t_{m}\right] \\
s_{1}=v_{0} \cdot t+\frac{1}{6} j_{\text {max }} \cdot t^{3}
\end{array}\right.
$$




$$
\begin{aligned}
& a_{2}=j_{\text {max }} \cdot t_{m}-j_{\text {max }} \cdot\left(t-t_{m}\right) \\
& \left\{v_{2}=v_{1}+j_{\text {max }} \cdot t_{m} \cdot\left(t-t_{m}\right)-\frac{1}{2} j_{\text {max }} \cdot\left(t-t_{m}\right)^{2} \quad, \quad t \in\left[t_{m}, 2 t_{m}\right]\right. \\
& s_{2}=s_{1}+v_{1} \cdot\left(t-t_{m}\right)+\frac{1}{2} j_{\text {max }} \cdot t_{m} \cdot\left(t-t_{m}\right)^{2}-\frac{1}{6} j_{\max } \cdot\left(t-t_{m}\right)^{3} \\
& \left\{a_{3}=0\right. \\
& \left\{v_{3}=v_{1}+\frac{1}{2} \cdot j_{\max } \cdot\left(t_{m}\right)^{2} \quad, t \in\left[2 t_{m}, 2 t_{m}+t_{c}\right]\right. \\
& s_{3}=s_{2}+\left[v_{1}+\frac{1}{2} \cdot j_{\text {max }} \cdot\left(t_{m}\right)^{2}\right] \cdot\left(t-2 t_{m}\right) \\
& \left\{\begin{array}{l}
a_{4}=-j_{\text {max }} \cdot\left[t-\left(2 t_{m}+t_{c}\right)\right] \\
v_{4}=v_{3}-\frac{1}{2} j_{\text {max }} \cdot\left[t-\left(2 t_{m}+t_{c}\right)\right]^{2} \\
s_{4}=s_{3}+v_{3} \cdot\left[t-\left(2 t_{m}+t_{c}\right)\right]-\frac{1}{6} j_{\max } \cdot\left[t-\left(2 t_{m}+t_{c}\right)\right]^{3}
\end{array}, \quad t \in\left[2 t_{m}+t_{c}, 3 t_{m}+t_{c}\right]\right. \\
& a_{5}=-j_{\text {max }} \cdot t_{m}+j_{\text {max }} \cdot\left[t-\left(3 t_{m}+t_{c}\right)\right] \\
& \left\{v_{5}=v_{4}-j_{\text {max }} \cdot t_{m} \cdot\left[t-\left(3 t_{m}+t_{c}\right)\right]+\frac{1}{2} j_{\text {max }} \cdot\left[t-\left(3 t_{m}+t_{c}\right)\right]^{2} \quad, t \in\left[3 t_{m}+t_{c}, 4 t_{m}+t_{c}\right]\right. \\
& s_{5}=s_{4}+v_{4} \cdot\left[t-\left(3 t_{m}+t_{c}\right)\right]-\frac{1}{2} j_{\max } \cdot t_{m} \cdot\left[t-\left(3 t_{m}+t_{c}\right)\right]^{2}+\frac{1}{6} j_{\max } \cdot\left[t-\left(3 t_{m}+t_{c}\right)\right]^{3}
\end{aligned}
$$

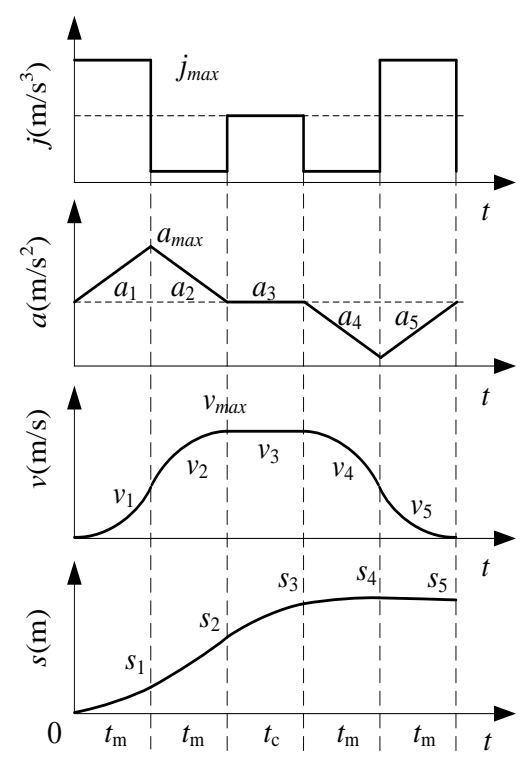

Figure 4. 5 segments acc/dec Curve in Long Motion Distance 


\subsection{Establishment of Modified Acc/Dec Curve}

When the movement distance is fairly short, because of the acceleration and velocity cannot reach the maximum, and the motion lacks the buffer function in uniform motion segments, the residual impact and vibration caused by the changes in acceleration of inflection point is more obvious than in long distance movement. So the method of 5 segments acc/dec is not suitable for short distance movement. Hence, modified acc/dec curve is proposed for the robot in short distance movement. In this method, jerk changes in a linear fashion, and acceleration segment is connected with deceleration segment; jerk is continuous, and the acceleration is quadratic function curve with continuous and smooth, which ensure the smooth start-stop movement of the robot. Meanwhile the algorithm involves less parameters and segments with easy computing and programming. The establish jerk, velocity, acc/dec and displacement are shown in Figure 5.

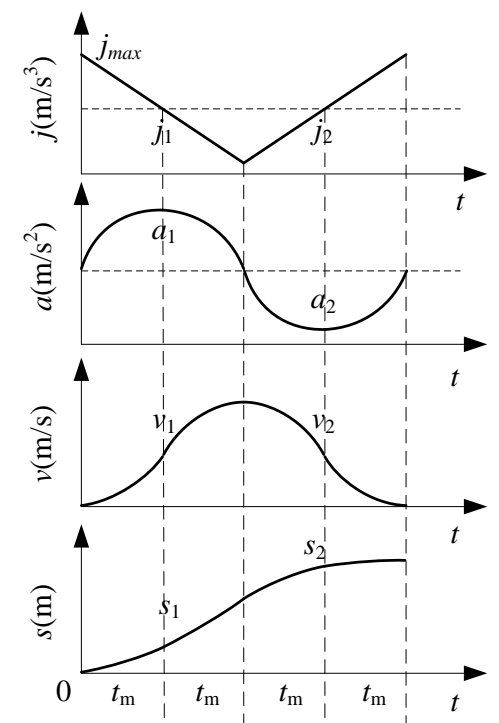

Figure 5. Modified acc/dec Curve in Short Motion Distance

The expressions of modified acc/dec are shown in Equations (11)-(12). Where, the definition of $t_{\mathrm{m}}$ is the same as in pure $\mathrm{S}$ acc/dec curve. The form of acc/dec can be determined by confirming the time parameter $t_{\mathrm{m}}$ only.

$$
\left\{\begin{array}{l}
j_{1}=-\frac{j_{\mathrm{max}}}{t_{m}} \cdot t+j_{\mathrm{max}} \\
a_{1}=a_{0}-\frac{j_{\mathrm{max}}}{2 t_{m}} \cdot t^{2}+j_{\mathrm{max}} \cdot t \\
v_{1}=v_{0}+a_{0} \cdot t-\frac{j_{\mathrm{max}}}{6 t_{m}} \cdot t^{3}+\frac{1}{2} j_{\mathrm{max}} \cdot t^{2} \\
s_{1}=s_{0}+v_{0} \cdot t+\frac{1}{2} a_{0} \cdot t^{2}-\frac{j_{\mathrm{max}}}{24 t_{m}} \cdot t^{4}+\frac{1}{6} j_{\max } \cdot t^{3}
\end{array}, t \in\left[0,2 t_{m}\right]\right.
$$




$$
\left\{\begin{array}{l}
j_{2}=\frac{j_{\mathrm{max}}}{t_{m}} \cdot\left(t-2 t_{m}\right)-j_{\mathrm{max}} \\
a_{2}=a_{1}+\frac{j_{\mathrm{max}}}{2 t_{m}} \cdot\left(t-2 t_{m}\right)^{2}-j_{\mathrm{max}} \cdot\left(t-2 t_{m}\right) \\
v_{2}=v_{1}+a_{1} \cdot\left(t-2 t_{m}\right)+\frac{j_{\mathrm{max}}}{6 t_{m}} \cdot\left(t-2 t_{m}\right)^{3}-\frac{1}{2} j_{\mathrm{max}} \cdot\left(t-2 t_{m}\right)^{2} \quad, \quad t \in\left[2 t_{m}, 4 t_{m}\right] \\
s_{2}=s_{1}+v_{1} \cdot\left(t-2 t_{m}\right)+\frac{1}{2} a_{1} \cdot\left(t-2 t_{m}\right)^{2}+\frac{j_{\mathrm{max}}}{24 t_{m}} \cdot\left(t-2 t_{m}\right)^{4}-\frac{1}{6} j_{\max } \cdot\left(t-2 t_{m}\right)^{3}
\end{array}\right.
$$

\subsection{Piecewise Self-adjusting Distribution of Acc/Dec}

Due to the differences of oral parameters between patients, the position information of the control nods planned differs from one to another. Therefore each of the movement distance in processing is different and each patient oral parameter should generate control its nods planning and acc/dec planning. In the meantime, despite in one patient, the control nods are not distributed uniformly, and the distance between control nods are not same. As a consequence, the piecewise self-adjusting distribution of acc/dec has double meanings.

By real-time comparing the relationship of the standard distance $s^{*}$ between the planning theory and robot system platform, and then distribute corresponding acc/dec curve between adjacent two control points, the residual vibration and shock can be minimized in the process of movement. If the movement distance is longer than the displacement of pure $\mathrm{S}$ curve, 5 segments $\mathrm{S}$ acc/dec curve is adopted, or else $\mathrm{S}$ acc/dec curve is adopted. The piecewise self-adjusting distribution of acc/dec is shown in Figure 6.

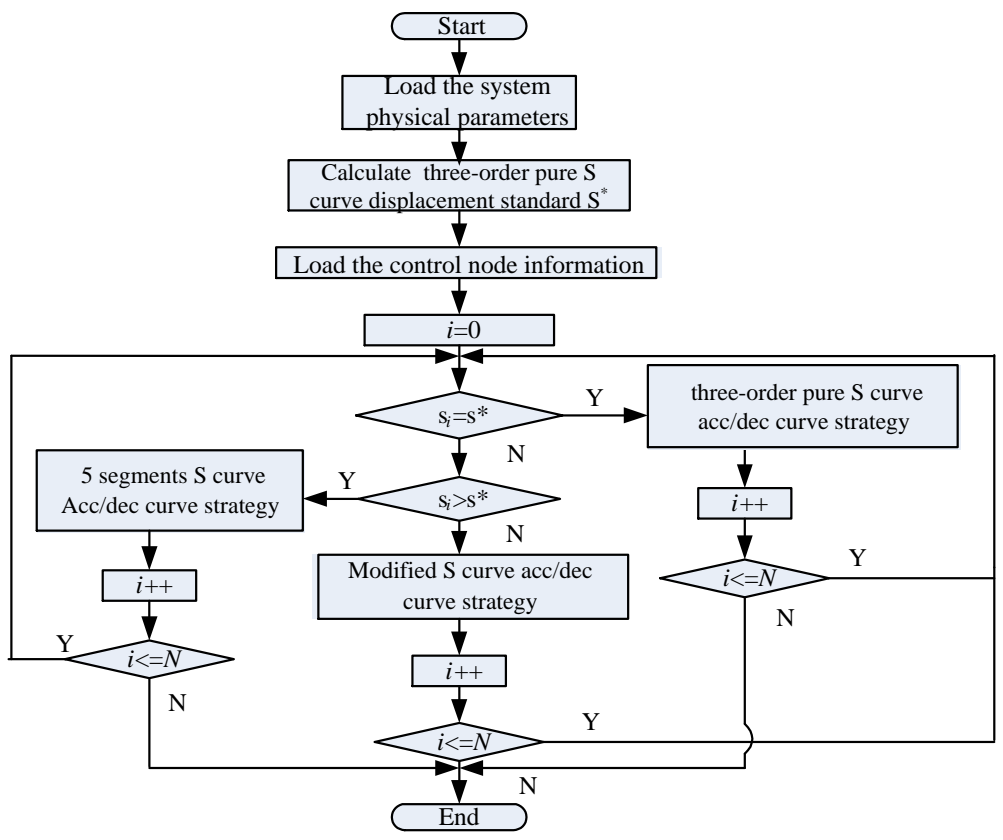

Figure 6. Piecewise Self-adjusting Distribution of acc/dec 


\section{Algorithm Simulation}

In order to realize validity of the motion planning, we use MATLAB to simulate different lengths in two period of movement distance of acc/dec. The Robot system platform parameters are shown as follows: $j_{\max }=40 \mathrm{~mm} / \mathrm{s}^{3}, a_{\max }=10 \mathrm{~mm} / \mathrm{s}^{2}, v_{\max }=2.5 \mathrm{~mm} / \mathrm{s}, v_{0}=v_{\mathrm{e}}=0$. After calculation, we get the corresponding movement distance at this time is $1.25 \mathrm{~mm}$. Therefore, we take the movement distance of pure $\mathrm{S}$ curve as a criterion, and assume two movement distance are $2.5 \mathrm{~mm}$ and $0.625 \mathrm{~mm}$. The parameters of the two movement distance by the planning of 5 segments acc/dec curve and modified pure $S$ acc/dec curve are shown in Table 1.In this table, the parameter of cts represents the quantity of pulse the drivers received. The acc/dec curves planned in two kinds of movement distance are shown in Figure 7 and Figure 8.

Table 1. Results Parameters of acc/dec Planned

\begin{tabular}{c|c|c}
\hline Parameters & Long distance motion & Short distance motion \\
\hline Motion distance (cts) & 4000 & 1000 \\
\hline$t_{\mathrm{m}}(\mathrm{s})$ & 0.25 & 0.2271 \\
\hline$t_{\mathrm{c}}(\mathrm{s})$ & 0.5 & None \\
\hline Maximum speed (cts/s) & 4000 & 2201 \\
\hline Maximum acceleration $(\mathrm{cts} / \mathrm{s})$ & 16000 & 7268 \\
\hline
\end{tabular}

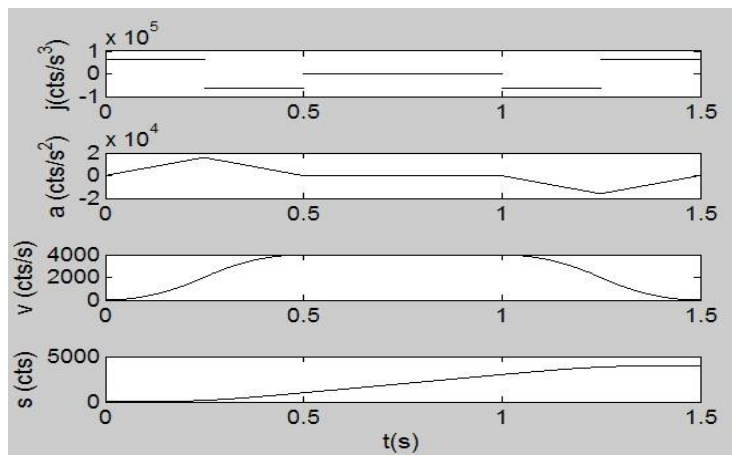

Figure 7. Simulation Curve of 5 Segments acc/dec

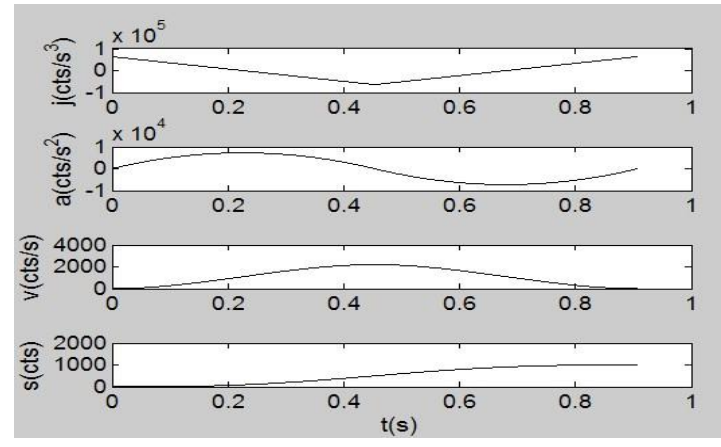

Figure 8. Simulation Curve of Modified S acc/dec 
According to Table 1, Figure 7 and Figure 8, the values of velocity and acceleration in long distance motion all reached the maximum, while short distance motion does not reach the maximum; the acceleration changes in a quadratic fashion, and the curve is smooth, so the flexible is better. As to the running time, the time will become longer by the jerk changes in a linear fashion in short distance movement, therefore despite the distance in long movement is three times than in short distance, the running time is basically the same, that is to say, increase flexible motion by sacrificing running time. And the simulation results are consistent with the analysis above.

\section{Conclusion}

In this paper, a new motion planning method based on piecewise self-adjusting distribution strategy of acc/dec is proposed and realized by the MATLAB simulation. Pure $\mathrm{S}$ curve is taken as a criterion and 5 segments $\mathrm{S}$ acc/dec curve and modified $\mathrm{S}$ acc/dec curve is adopted, which can ensure the robot in each control nod with high efficiency steady movement and the feasibility of motion planning is proved..

\section{Acknowledgements}

This research was supported by the National High Technology Research and Development Program of China ('863' Program) (Nos. 2013AA040803), and by National Natural Science Funds of China (Grant Nos. 51205093).

\section{References}

[1] Y. Zhang and J. Jiang, "Bending Property Analysis and Experimental Study of Orthodontic Wires", vol. 22, no. 15, (2011), pp. 1827-1831.

[2] Z. Xiao and J. Nie, "Research and Comparison for Acceleration and Deceleration Control Algorithm", International Conference of Information Technology, Computer Engineering and Management Sciences, (2011); China.

[3] K. Zhou and Q. Lu, "The Flexible Acceleration and Deceleration Control Method of CNC Machine Tools" vol. 40, no. 8, pp.39-41, (1999).

[4] L. Lu and H. Pan, "Trajectory Planning for Point-to-point Movement with a Constant Velocity Duration", vol. 47, no. 5, (2006), pp. 8-11.

[5] P.H. Meckl, P.B. Arestides, M.C. Woods, "Optimized S-curve motion profiles for minimum residual vibration", The American Control Conference, (1998); USA.

[6] C. Ruo, C.-L. Shih and W.-Y. Lee, "Planning S-curves in the coordinated PTP motion of multiple-axis machine under velocity acceleration and jerk constraints", (2003), vol. 10, no. 3, pp. 221-234.

[7] X. Shi, B. Xu, W. Xie and B. Li., "Design and Implementation of S-shape Acceleration/Deceleration Algorithm Based on Rounding Error Compensation Tactic", The World Congress on Intelligent Control and Automation, (2008); China.

[8] L. Hu, S. Lin, J. Xu and H. Dong, "A New Speed Control Algorithm for S-curve Acceleration Deceleration”, (2010), vol. 51, no. 1, pp. 22-27.

[9] H. Pan, Y. Wei, L. Chen, H. Tan and H. Sun, "Adaptive Piecewise NURBS Curve Interpolator Algorithm for Entireness Process S-curve ACC/DEC Control”, vol. 21, no. 2, (2011), pp. 190-195.

[10] G. Chen and X. Mei, "A Dissymmetrical S Curve Velocity Plan \& Look-ahead Algorithm for Small Line Segments", vol. 47, no. 8, (2010), pp. 47-49.

[11] S. Zhao, D. Yu, C. Geng and W. Han, "Research on Lookahead Interpolation Algorithm Based on Cubic Polynomial Displacement Incremental Model”, vol. 24, no. 8, (2013), pp. 1066-1073.

[12] J. Tian, H. Lin, Z. Yao and J. Li, "Study on S-shape Curve Acceleration and Deceleration Control Fast Planning on CNC System”, (2013), vol. 34, no. 1, pp. 168-172.

[13] D. Du, Y. Liu, X. Guo, K. Yamazaki and M. Fujishima, "An accurate adaptive NURBS curve interpolator with real-time flexible acceleration/deceleration control”, (2010), vol. 26, no. 4, pp. 273-281.

[14] G. Ji, "Study on NURBS Curve Interpolation Based on before Acceleration/Deceleration with Linear Jerk", 3th International Conference on Advances in Materials and Manufacturing Processes, (2013); China. 
[15] H. Fu, "NURBS interpolator with flexible acceleration and deceleration based on curvature properties", vol. 18, no. 9, (2012), pp. 1921-1929.

[16] K.-H. Rew and K.-S. Kim, "A closed-form solution to asymmetric motion profile allowing acceleration manipulation", IEEE Transactions on Industrial Electronics, (2010).

[17] J. W. Jeon, "Efficient acceleration and deceleration technique for short distance movement in industrial robots and CNC machine tools", vol. 8, (2000), pp. 766-768. 
International Journal of Control and Automation Vol.7, No.7 (2014) 